\title{
Vaccination-A Step Closer to Universal Health Coverage
}

\author{
Narges Kalantari ${ }^{1,2} \cdot$ Bettina Borisch ${ }^{1,3} \cdot$ Marta Lomazzi $^{1,3}$ (D)
}

Received: 6 March 2020 / Accepted: 16 May 2020 / Published online: 20 June 2020

(C) Springer-Verlag GmbH Germany, part of Springer Nature 2020

\begin{abstract}
Background Vaccination saves countless lives worldwide. However, a gap in accessibility to and acceptability of vaccines is observed locally and globally. This gap must be addressed through a combined intersectoral approach that takes into account different social, economic, political and environmental aspects of vaccination. In addition, a comprehensive strategy is necessary to provide better immunization to all as the Decade of Vaccines comes to an end.

Methods One of the objectives of the World Health Organization (WHO) is to provide equitable access to quality vaccination around the world. Economic barriers as well as conflict situations lower vaccination coverage and hinder reaching the objective of having an immunization-for-all status. When resources are limited, innovative approaches to supplying vaccines are crucial for facing the challenges of our time and reaching this objective. Furthermore, an active demand for vaccination must be developed through advocacy, knowledge generation and building trust. A positive environment must be created around vaccination to reduce vaccine hesitancy and increase vaccination uptake. To increase vaccine accessibility and acceptability, the World Federation of Public Health Associations (WFPHA) developed a Call to Action based on the Global Charter for the Public's Health, which demands all actors to collaborate in creating strong health policies for vaccination.

Conclusions An intersectoral approach is required to increase the accessibility and acceptability of vaccines. The WFPHA's Call to Action proposes strategies to reach better immunization outcomes using the Global Charter as a platform for developing better vaccination policies.
\end{abstract}

Keywords Vaccination · Global charter $\cdot$ Global public health $\cdot$ Universal health coverage

\section{Introduction}

Every year, many lives worldwide are saved by vaccination. Since its establishment in 1974, the Expanded Programme on Immunization (EPI) has increased immunization coverage for its six initial targeted diseases (measles, diphtheria, pertussis, tetanus, poliomyelitis and tuberculosis) from $5 \%$ to $86 \%$ and even $>95 \%$ in certain developing countries (WHO 2013a, 2017a). Despite this progress, the world has significant deficiencies in immunization. The 2018 assessment report of the Global Vaccine Action Plan (GVAP) showed that measles

Marta Lomazzi

Marta.Lomazzi@unige.ch

1 World Federation of Public Health Associations (WFPHA), ch. des Mines, 1202 Geneva, Switzerland

2 Psychology Department, University of Montreal, 90, avenue Vincent d'Indy, Montreal, QC, Canada

3 Institute of Global Health, University of Geneva, 9 ch. des Mines, 1202 Geneva, Switzerland and diphtheria outbreaks still occur in many WHO regions, 19.9 million children are insufficiently immunized, and only very few countries have stated no vaccine hesitancy (WHO 2018a). Because health has multiple social, political, environmental and economic determinants, these issues require attention from different sectors to develop good policies (WHO 2016). A combined effort toward immunization is in line with the Alma-Ata declaration of 1978 that called for the "coordinated efforts of all related sectors in addition to the health sector" in delivering primary health care.

In addition to intersectoral action, a comprehensive approach is necessary to reach immunization for all as the Decade of Vaccines comes to an end. This approach can be inspired by the Global Charter for the Public's Health (Charter), which was developed by the World Federation of Public Health Associations (WFPHA) in collaboration with the World Health Organization (WHO). The Charter was developed in 2015-2016 through (1) numerous consultations with actors in public health and related sectors using a Delphi-like method (Gordon 1994); (2) examining the current public health models in different countries and regions such as 
the USA, UK, Australia and Canada; and (3) conducting a literature review on the terms "global health" and "global public health" (see Jenkins et al. 2016; Lomazzi et al. 2016). The Charter provides a framework to increase the effectiveness of public health at the local and global levels. It regards governance, advocacy, capacity and information as the four enablers of public health services, which include health promotion, disease prevention and protection of the public's health (Borisch et al. 2018; Lomazzi 2016; Moore et al. 2016). Under the Charter, the WFPHA has developed a Call to Action demanding a combined effort from all actors at the local and global level to improve accessibility and acceptability of cost-effective vaccines to protect the public's health (WFPHA 2019).

Inspirited by the enabler functions and the services of the Charter, this article aims to share knowledge on how to develop and strengthen immunization policies that can be applied at the local level while taking advantage of different global inputs. A discussion on innovation, coverage and equity as well as advocacy, information and trust is deemed necessary to develop immunization policies for better accessibility and acceptance of vaccines and taking a step closer to Universal Health Coverage, when "all people and communities can use the promotive, preventive, curative, rehabilitative and palliative health services they need, of sufficient quality to be effective, while also ensuring that the use of these services does not expose the user to financial hardship" (WHO 2020). This article provides a commentary on factors that are deemed important for better vaccination outcomes based on the WFPHA's policy experience and advocacy dialogue with key organizations. Selected examples of how the Charter services and enabler functions can be implemented within this context are highlighted in the text, and a broad overview is given in Fig. 1.

\section{Access through innovation, expanded coverage and enhanced equity}

Worldwide equitable access to vaccination is one of the objectives of the WHO and the Global Alliance for Vaccines and Immunization (GAVI) (GAVI 2019b; WHO 2018a). Nevertheless, a large gap exists in access to vaccines at the national and international levels. Factors such as conflict and low financial resources in a country can lower the immunization coverage (WHO 2018a) and threaten vaccine equity. At times of conflict, the resettlement of displaced populations in overcrowded camps with unsanitary conditions brings even more health challenges for the host country. This has been the case in Rohingya refugee camps in Bangladesh where serious diphtheria outbreaks started at the end of 2017 (Rahman and Islam 2019). Moreover, an expected 13\% increase in the urban population of the world by 2050 (UN 2018) requires more innovative ways to overcome the new health challenges of overcrowded urban environments and to provide vaccination to all (GAVI 2019a). Investing in developing innovative approaches to research and development (R\&D) and increasing supplies to improve equitable access to vaccines are certainly even more crucial in such conditions.

It is not surprising to observe shortages in the supply of vaccines when demands are high while resources are limited. For example, reaching the objective of eliminating cervical cancer is challenged by the anticipated three-fold increase in demand for human papillomavirus (HPV) vaccines within the next 10 years (WHO 2018b). Therefore, programs such as Innovation for Uptake, Scale and Equity in Immunization (INFUSE) launched by GAVI are essential so that the best innovations can be "infused" with resources to accelerate providing more quality vaccines (GAVI 2019a). The efforts of the Coalition for Epidemic Preparedness Innovations (CEPI) to advance R\&D through investing in "vaccine platform technologies," identify and support new technologies for delivery and uptake of vaccines, and engage in coordinated efforts between different actors to test and distribute vaccines are also essential in this endeavor (CEPI 2018). Furthermore, the Charter service of "prevention" is key to develop and strengthen such intersectoral collaborations to lead innovative research according to real needs and to commit appropriate funding. This is ony possible through sustainable commitments by governments to optimize the protection of the public's health through investment in vaccination and adoption of best practices, thus guaranteeing sufficient and equitable access to vaccines.

Similarly, the "knowledge generation" and "capacity building" enablers of the Charter are important in "equitably extending the benefits of immunization to all people," which is listed as the third strategic objective of the GVAP (WHO 2013b). Hence, the WFPHA calls for empowering the health workforce by supporting research and communication, improving the knowledge of health professionals and leaders through training and education, improving infrastructure by providing accountable data and electronic immunization registers as well as supporting vaccination campaigns (WFPHA 2019). Committing to advocacy to strengthen health systems and improve human resources for health is equally important for increasing the workforce capacity and ensuring equitable access to vaccination. However, another crucial element in expanding coverage is to avoid making new boundaries to immunization between different countries. The transition from low to middle-income status may result in disqualification for receiving international aid (UNICEF 2018), which can serve as a barrier to vaccination. This is remarkable as middleincome countries that are not supported by GAVI are not doing as well as expected in terms of immunization (WHO 2018a), which challenges accomplishing the Charter's service of "health protection." In this regard, the use of differential pricing to set price tiers and pooled procurement methods, in 


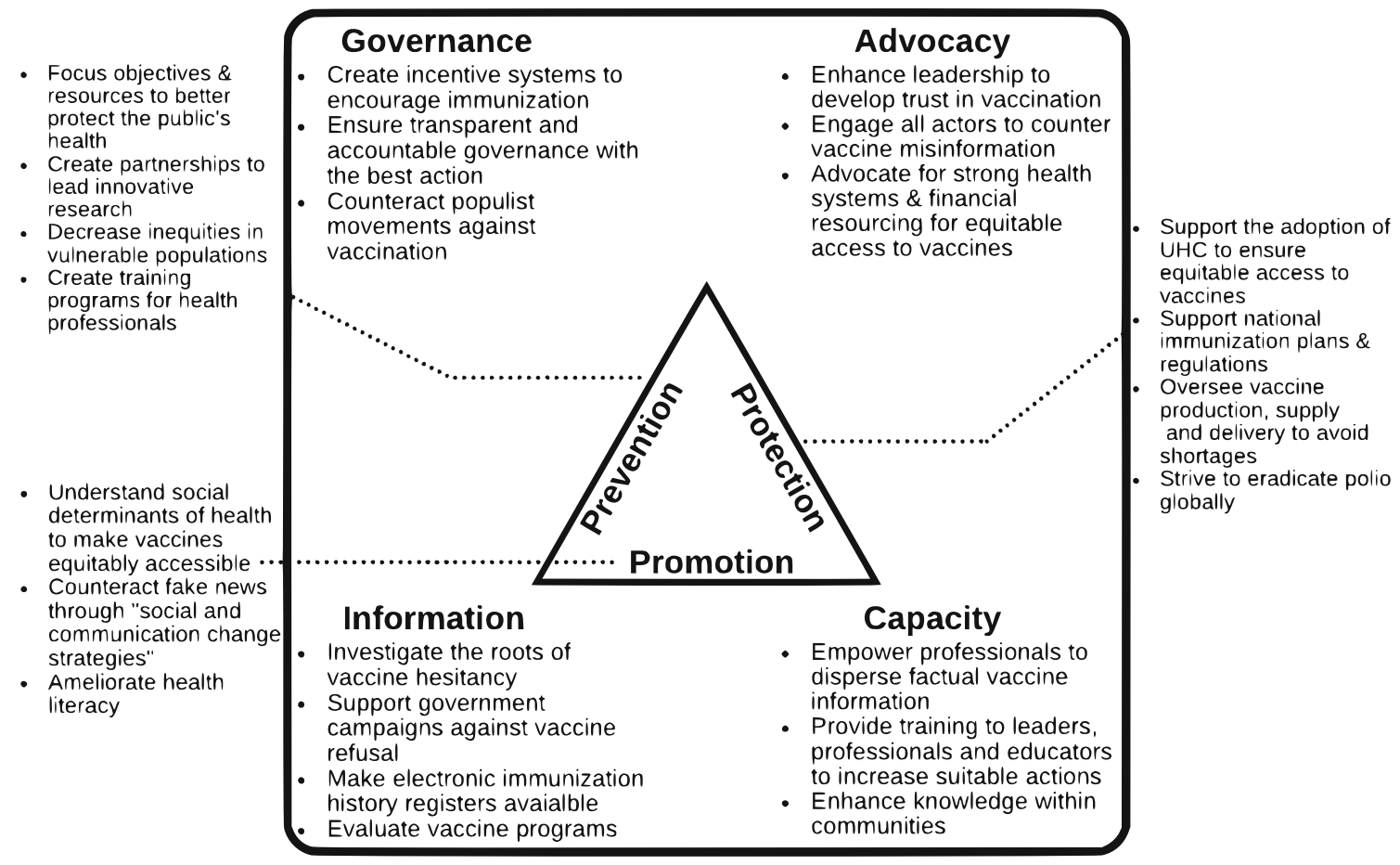

Fig. 1. Improving vaccination through the Charter's enablers and services. The enabler functions of the Charter (Information, Capacity, Governance and Advocacy) in parallel with the public health services

addition to increasing the total funding available for vaccination, are crucial to ensure vaccine affordability in lowermiddle-income and middle-income countries (WHO 2013b).

\section{Acceptance through Advocacy, Knowledge Generation and Creating Trust}

Expanding vaccine production and availability alone is not sufficient to reach coverage. The value of vaccines must be understood at both the individual and community level, and active demand must be created through community involvement, accountability and response to unfavorable incidents and challenges (WHO 2018a). This engagement will allow building a positive environment for vaccination, which is crucial in reducing vaccine hesitancy (WHO 2018a), a situation that was regarded as one of the ten threats to global health in 2019 (WHO 2019) and that refers to a "delay in acceptance or refusal of vaccination despite availability of vaccination services" (MacDonald and SAGE Working Group on Vaccine Hesitancy 2015). A survey from 67 countries in 2016 reported that, on average, $13 \%$ of individuals declared mistrust in vaccination safety. This is particularly remarkable in Europe: In France and Bosnia-Herzegovina, $41 \%$ and $36 \%$ of survey participants perceived vaccines as unsafe, respectively (Larson et al. 2016). Moreover, in Italy, a country that ranked second in the prevalence of measles in the European Union (EU) in 2017 (ECDC 2018), 15.4\% of participants expressed skepticism toward vaccination importance (Larson et al. 2016). An of health promotion, protection and disease prevention are essential to improving the state of vaccination in the world. This figure shows how these elements come together to increase vaccine availability and uptake

example of the vaccine hesitancy issue outside the EU is the case of South Africa where cervical cancer is the second most prevalent type of cancer in women (GLOBOCAN 2018) and where hesitancy is turning into an additional challenge to the success of the school-based HPV immunization program (Ngcobo et al. 2018).

A growing number of individuals have become vaccinehesitant because of the risk-benefit concerns that are raised related to political motives and social media (WHO 2018a). Because of its negative influence on vaccination confidence, the availability of misinformation on social media should be identified as a threat to the public's health (Larson 2018). For this reason and in agreement with the Charter enabler of "accurate information," the WFPHA calls for counteracting fake news through examining the "rationales and the emotions" behind vaccine hesitancy, spreading factual information on social media and supporting government campaigns against vaccine refusal (WFPHA 2019). Political forces such as populist movements that exploit vaccination as a means to gain approval should also be taken into account. In addition, social media platforms must proactively fight against fake news. Facebook, Pinterest, YouTube and Amazon have recently joined the efforts against vaccine hesitancy by filtering vaccine misinformation and advertisements (Bickert 2019; Zadrozny 2019). Although this is an important step toward tackling fake news about vaccines, it must be expanded to other online health misinformation to improve the availability of accurate information, promote health and prevent diseases. In a vaccine-hesitant atmosphere, 
health education and advocacy are essential in changing attitudes. Nevertheless, they must go beyond providing scientific information; each opportunity must be used to create a warm and positive interaction between the health workforce and the users to understand the roots of the issue. Building relationships based on trust between stakeholders and communities through competence, objectivity, fairness, consistency, sincerity and goodwill (Renn 2008) as well as providing support in times of crises is crucial to avoid misperceptions and skepticism (WHO 2017b).

The reasons behind vaccine hesitancy are highly variable and context-specific (Dubé et al. 2013). Therefore, parallel with the Charter function of "health promotion," "addressing the causes of the causes" of vaccine hesitancy is fundamental to understanding the socio-cultural barriers that affect confidence in vaccination (WFPHA 2019). Furthermore, it is important to combine advocacy and knowledge generation with good policies that reinforce good practices such as the approval of law 119/2017 in Italy that made vaccinations mandatory for children (Scavone et al. 2018; Signorelli et al. 2017). This was followed by progress toward higher vaccination coverage in Italy (D'Ancona et al. 2019; Signorelli et al. 2018). Aligned with the Charter enabler function of "good governance" for the protection of the public's health, legislations to reinforce good health practices must be in place. Moreover, scientific knowledge and political actions must be strongly linked so that only accurate, univocal and unbiased messages are distributed to the public (Ricciardi and Siliquini 2019). It is also crucial to highlight the importance of the attitudes and actions of governments toward vaccination as only one wrong step may result in failure to vaccinate. This has been the case of HPV vaccines in Japan, where the government suspended the HVP immunization recommendation in 2013 because of misinformation that was spread through the media. This action resulted in distrust in HPV vaccination within and beyond the borders of Japan (Larson et al. 2014). This example illustrates how governments play a notable role in influencing the attitudes toward vaccination, the success of immunization uptake and consequently the public's health.

\section{Conclusion}

An intersectoral approach engaging actors from public health, health-related professions and governments is required to achieve better immunization outcomes, which are considered "the central pillar of the Universal Health Coverage" (WHO 2018a). The WFPHA provides comprehensive strategies through the enablers and services of the Global Charter for the Public's Health and emphasizes the importance of knowledge transfer and exchange of best practices between different countries and organizations as well as the political aspect of health actions. The WFPHA's Call to Action, which is rooted in the Charter and is aligned with the six strategic objectives of
GVAP (WHO 2013b), supports moving toward the Universal Health Coverage by advocating for quality vaccines for all and encourages "all levels of governments, governance levels in corporations (GAFAs), public health associations and likeminded organizations in every country to prioritize evidence-based immunization decision-making" to increase the supplies of vaccines, raise awareness and strengthen capacity to protect the public's health (WFPHA 2019). Better vaccination outcomes can be reached if only accurate information, improved advocacy, good governance and strengthened capacity are combined through a joint global effort to promote health, prevent diseases and protect the public's health. These efforts must be adaptive and consider the new challenges of our time that are raised because of the displacement of populations from conflict zones, rapid urbanization that leaves vulnerable populations unnoticed, significant increases in demands that exceed supplies, rising inequity worldwide and the spread of fake news that results in distrust and reemergence of eradicated vaccine-preventable diseases.

Acknowledgements Not applicable

Authors' Contributions ML conceived the idea of this manuscript. NK conducted literature research and wrote the manuscript. NK, ML and BB participated in its revision. All authors read and approved the manuscript.

Funding The funding comes from the WFPHA general budget. The funders of this research did not contribute to study design development, analysis, interpretation of data or writing the manuscript.

Availability of Data and Materials Not applicable

\section{Compliance with ethical standards}

Ethics Approval and Consent to Participate: Not applicable

Consent for Publication Not applicable

Competing Interests The authors declare that they have no competing interests.

Abbreviations CEPI, Coalition for Epidemic Preparedness Innovations; Charter, Global Charter for the Public's Health; EPI, Expanded Programme on Immunization; EU, European Union; GAFA, Google, Apple, Facebook, Amazon; GAVI, Global Alliance for Vaccines and Immunization; GVAP, Global Vaccine Action Plan; HPV, human papillomavirus; INFUSE, Innovation for Uptake, Scale and Equity in Immunization; R\&D, Research and Development; WFPHA, World Federation of Public Health Association; WHO, World Health Organization

\section{References}

Bickert M (2019) Combatting Vaccine Misinformation. Facebook. https://newsroom.fb.com/news/2019/03/combatting-vaccinemisinformation/. Accessed 13 August 2019 
Borisch B, Lomazzi M, Moore M, Krech R (2018) Update on the Global Charter for the Public's Health. Bull World Health Organ 96:439 440. https://doi.org/10.2471/BLT.17.198820

CEPI (2018) CEPI Progress Report. The Coalition for Epidemic Preparedness Innovations https://cepi.net/wp-content/uploads/ 2019/05/CEPI-Progress-Report_2018.pdf. Accessed 5 August 2019

D’Ancona F, D'Amario C, Maraglino F, Rezza G, Iannazzo S (2019) The law on compulsory vaccination in Italy: an update 2 years after the introduction. Eurosurveillance 24:1900371. https://doi.org/10.2807/ 1560-7917.ES.2019.24.26.1900371

Dubé E, Laberge C, Guay M, Bramadat P, Roy R, Bettinger JA (2013) Vaccine hesitancy: an overview. Human vaccines \& immunotherapeutics 9:1763-1773. https://doi.org/10.4161/hv.24657

ECDC (2018) Measles outbreaks still ongoing in 2018 and fatalities reported from four countries. European Centre for Disease Prevention and Control. https://ecdc.europa.eu/en/news-events/measlesoutbreaks-still-ongoing-2018-and-fatalities-reported-four-countries. Accessed 3 July 2019

GAVI (2019a) 2019 INFUSE Call for Innovation. https://www.gavi.org/ investing/infuse/calls-to-innovate/2019/. Accessed 10 July 2019

GAVI (2019b) Health equity. Gavi The Vaccine Alliance https://www. gavi.org/about/value/health-equity/. Accessed 5 August 2019

GLOBOCAN (2018) Cancer Today. World Health Organization International Agency for Research on Cancer http://gco.iarc.fr/ today/home. Accessed 8 August 2019

Gordon TJ (1994) The Delphi method. Futures research methodology 2:1-30

Jenkins C, Lomazzi M, Yeatman H, Borisch B (2016) Global public health: a review and discussion of the concepts, principles and roles of global public health in Today's society. Global Policy 7:332-339. https://doi.org/10.1111/1758-5899.12302

Larson HJ (2018) The biggest pandemic risk? Viral misinformation. Nature 562:309. https://doi.org/10.1038/d41586-018-07034-4

Larson HJ et al (2016) The State of Vaccine Confidence 2016: Global insights through a 14-country survey. EBioMedicine 12:295-301. https://doi.org/10.1016/j.ebiom.2016.08.042

Larson HJ, Wilson R, Hanley S, Parys A, Paterson P (2014) Tracking the global spread of vaccine sentiments: the global response to Japan's suspension of its HPV vaccine recommendation. Human vaccines \& immunotherapeutics 10:2543-2550. https://doi.org/10.4161/ 21645515.2014.969618

Lomazzi M (2016) A Global Charter for the Public's Health-the public health system: role, functions, competencies and education requirements. Eur J Public Health 26:210-212. https://doi.org/10.1093/ eurpub/ckw011

Lomazzi M, Jenkins C, Borisch B (2016) Global public health today: connecting the dots. Global Health Action 9. https://doi.org/10. 3402/gha.v9.28772

NE MD, SAGE Working Group on Vaccine Hesitancy (2015) Vaccine hesitancy: Definition, scope and determinants. Vaccine 33:41614164. https://doi.org/10.1016/j.vaccine.2015.04.036

Moore M, McKee M, Borisch B, Ricciardi W (2016) The Global Charter for the Public's Health. European Journal of Public Health 26:207. https://doi.org/10.1093/eurpub/ckw013

Ngcobo NJ, Burnett RJ, Cooper S, Wiysonge CS (2018) Human papillomavirus vaccination acceptance and hesitancy in South Africa: Research and policy agenda. South African Medical Journal 109: 13-15. https://doi.org/10.7196/SAMJ.2018.v109i1.13723

Rahman MR, Islam K (2019) Massive diphtheria outbreak among Rohingya refugees: lessons learnt. J Travel Med 26:tay122. https:// doi.org/10.1093/jtm/tay122

Renn O (2008) Risk communication: Insights and requirements for designing successful communication programs on health and environmental hazards. In: Heath RL, O'Hair HD (eds) Handbook of risk and crisis communication 1edn. Routledge, pp 81-99
Ricciardi W, Siliquini R (2019) Political determinants of health and vaccination. J Public Health Pol 40. https://doi.org/10.1057/s41271$019-00167-4$

Scavone C et al. (2018) Did the new Italian law on mandatory vaccines affect adverse event following immunization's reporting? A pharmacovigilance study in Southern Italy. Frontiers in pharmacology 9. https://doi.org/10.3389/fphar.2018.01003

Signorelli C, Guerra R, Siliquini R, Ricciardi W (2017) Italy's response to vaccine hesitancy: An innovative and cost-effective National Immunization Plan based on scientific evidence. Vaccine 35: 4057-4059. https://doi.org/10.1016/j.vaccine.2017.06.011

Signorelli C, Odone A, Cella P, Iannazzo S (2018) Childhood vaccine coverage in Italy after the new law on mandatory immunization. Ann Ig 1:1-10. https://doi.org/10.7416/ai.2018.2227

UN (2018) $68 \%$ of the world population projected to live in urban areas by 2050 , says UN. United Nations. https://www.un.org/ development/desa/en/news/population/2018-revision-of-worldurbanization-prospects.html. Accessed 23 July 2019

UNICEF (2018) UNICEF Immunization Roadmap: 2018-2030. United Nations Children's Fund. https://www.unicef.org/sites/default/files/ 2019-01/UNICEF Immunization Roadmap 2018.pdf. Accessed 7 July 2019

WFPHA (2019) WFPHA Vaccination and Capacity Workshop Call to Action. World Federation of Public Health Associations. https:// www.wfpha.org/images/Call_to_Action_Final2.pdf. Accessed 7 July 2019

WHO (2013a) The Expanded Programme on Immunization. World Health Organization. https://www.who.int/immunization/ programmes_systems/supply_chain/benefits_of_immunization/en/. Accessed 10 July 2019

WHO (2013b) Global vaccine action plan 2011-2020. World Health Organization. https://www.who.int/immunization/global_vaccine action_plan/GVAP_doc_2011_2020/en/. Accessed 18 July 2019

WHO (2016) Intersectoral action for health-experiences from small countries in the WHO European Region. http://www.euro.who. int/ data/assets/pdf file/0017/325322/ISA-Experiences-smallcountries-WHO-ER.pdf. Accessed 17 July 2019

WHO (2017a) Ten years in public health, 2007-2017: report by Dr Margaret Chan, Director-General, World Health Organization. https://apps.who.int/iris/bitstream/handle/10665/255355/ 9789241512442-eng.pdf. Accessed 18 July 2019

WHO (2017b) Vaccination and Trust. World Health Organization. http:// www.euro.who.int/_data/assets/pdf_file/0004/329647/Vaccinesand-trust.PDF?ua=1. Accessed 12 July 2019

WHO (2018a) 2018 Assessment report of the Global Vaccine Action Plan. https://www.who.int/immunization/global_vaccine_action plan/SAGE_GVAP_Assessment_Report_2018_EN.pdf?ua=1. Accessed 12 July 2019

WHO (2018b) Global Market Study- HPV. https:/www.who.int/ immunization/programmes_systems/procurement/v3p/platform/ module2/en/. 12 July 2019

WHO (2019) Ten threats to global health in 2019. World Health Organization. https:/www.who.int/emergencies/ten-threats-toglobal-health-in-2019. Accessed 12 July 2019

WHO (2020) What is health financing for universal coverage? Geneva: World Health Organization. http://www.who.int/health_financing/ universal coverage definition. Accessed 04 March 2020

Zadrozny B (2019) Amazon removes books promoting autism cures and vaccine misinformation. NBCNews. https://www.nbcnews.com/ tech/internet/amazon-removes-books-promoting-autism-curesvaccine-misinformation-n982576. Accessed 13 August 2019

Publisher's note Springer Nature remains neutral with regard to jurisdictional claims in published maps and institutional affiliations. 